\title{
Práctica docente y ciudadanía en educación superior
}

\author{
Teaching Practice and Citizenship in Higher Education
}

Prática docente e cidadania no ensino superior

\author{
David Jasso Velazquez \\ Universidad Autónoma de Zacatecas, México \\ dajass971@uaz.edu.mx \\ https://orcid.org/0000-0002-8289-150X \\ Sonia Villagrán Rueda \\ Universidad Autónoma de Zacatecas, México \\ soniavillagran@uaz.edu.mx \\ https://orcid.org/0000-0001-5389-574X \\ Mónica Rodríguez Ortiz \\ Universidad Autónoma de Zacatecas, México \\ monipsic2017@uaz.edu.mx \\ https://orcid.org/0000-001-8268-1193
}

\section{Resumen}

El objetivo del presente estudio es determinar el grado de ciudadanía en los estudiantes de educación superior de la Unidad Académica de Psicología de la Universidad Autónoma de Zacatecas (UAZ), incluyendo la responsabilidad democrática, el respeto a las diferencias humanas y la convivencia y paz. El método fue cuantitativo de alcance correlacional y diseño transversal. Se aplicó un instrumento de elaboración propia denominado "Cuestionario de práctica docente y ciudadanía" a 106 estudiantes de la UAZ. Entre los resultados se encontró que existe una cohesión de fuerte a moderada entre las variables de los ejes Práctica Docente y Ciudadanía, ya que se observó una correlación por encima de 0.234, con una significancia menor a 0.05 . Esto indica que si el docente se forma de manera 


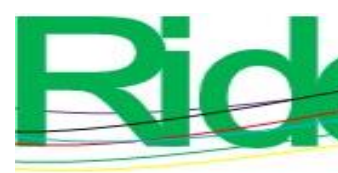

Revista Iberoamericana para la
Investigación y el Desarrollo Educativo
ISSN $2007-7467$

crítica, realiza valoraciones de los procesos de enseñanza y aprendizaje a través del diálogo, interactúa con otros docentes y construye y vive valores en su contexto educativo y social estará influyendo en la formación de un habitus ciudadano con su estudiantado, repercutiendo positivamente en la sociedad. Aquí se muestra que la docencia se encuentra totalmente vinculada a la conformación de prácticas de ciudadanía, lo que explica la relación contundente entre un docente preparado — que investiga, planifica y cuenta con una personalidad íntegra - y acciones de liderazgo de estudiantes capaces de solucionar y afrontar problemas y participar democráticamente.

Palabras clave: formación de docentes, enseñanza superior, liderazgo político.

\section{Abstract}

The objective of this study is to determine the degree of citizenship in higher education students of the Academic Unit of Psychology of the Universidad Autónoma de Zacatecas (UAZ), including democratic responsibility, respect for human differences and coexistence and peace. The method was quantitative with a correlational scope and a cross-sectional design. A self-made instrument called the "Teaching Practice and Citizenship Questionnaire" was applied to $106 \mathrm{UAZ}$ students. Among the results, it was found that there is a strong to moderate cohesion between the variables of the Teaching Practice and Citizenship axes, since a correlation was observed above 0.234 , with a significance lower than 0.05 . This indicates that if the teacher is trained in a critical way, makes assessments of the teaching and learning processes through dialogue, interacts with other teachers and builds and lives values in their educational and social context, they will be influencing the formation of a citizen habitus with its student body, having a positive impact on society. Here it is shown that teaching is totally linked to the formation of citizenship practices, which explains the strong relationship between a trained teacher — who investigates, plans and has an integral personality — and leadership actions of students capable of solving and facing problems and participating democratically.

Keywords: teacher education, university education, political leadership. 


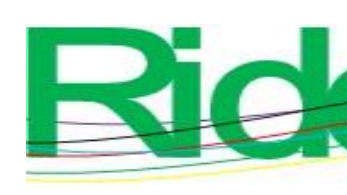

Revista Iberoamericana para la Investigación y el Desarrollo Educativo ISSN $2007-7467$

\section{Resumo}

O objetivo deste estudo é determinar o grau de cidadania em estudantes do ensino superior da Unidade de Psicologia Acadêmica da Universidade Autônoma de Zacatecas (UAZ), incluindo responsabilidade democrática, respeito às diferenças humanas e convivência e paz. O método foi quantitativo com escopo correlacional e delineamento transversal. Um instrumento de confecção própria denominado "Questionário de Prática de Ensino e Cidadania" foi aplicado a 106 alunos da UAZ. Entre os resultados, verificou-se que há coesão forte a moderada entre as variáveis dos eixos Prática de Ensino e Cidadania, visto que se observou correlação acima de 0,234, com significância inferior a 0,05. Isso indica que se o professor for formado criticamente, fizer avaliações dos processos de ensino e aprendizagem por meio do diálogo, interagir com outros professores e construir e viver valores em seu contexto educacional e social, estará influenciando na formação de um habitus cidadão. com seus alunos, impactando positivamente a sociedade. Aqui se mostra que a docência está totalmente ligada à formação de práticas cidadãs, o que explica a relação contundente entre um professor preparado - que investiga, planeja e tem uma personalidade sólida - e ações de liderança estudantil capazes de resolver e enfrentar problemas e participar democraticamente.

Palavras-chave: formação de professores, ensino superior, liderança política.

Fecha Recepción: Agosto 2021

Fecha Aceptación: Febrero 2022

\section{Introducción}

En las últimas décadas, en América Latina se ha estado dando un debate importante sobre la educación ciudadana, es decir, sobre la formación de competencias ciudadanas en los estudiantes de los diferentes niveles. Términos como ciudadanía, derechos humanos, valores, democracia, paz y necesidades de la sociedad aparecen ligados a la formación académica. Sin embargo, en la práctica estos aspectos trascendentales raramente son tenidos en consideración por el profesorado, al menos de una forma intencional y sistemática, a lo largo y ancho de su actividad docente. De hecho, en la educación superior se alcanza a apreciar que las prácticas docentes, delimitadas en gran medida por el currículo, no dan respuesta al tipo de formación integral que requiere el sujeto ni al fortalecimiento de los enfoques humanistas y ambientales (Organización de las Naciones Unidas para la Educación, la Ciencia y la Cultura [Unesco], 2017). 


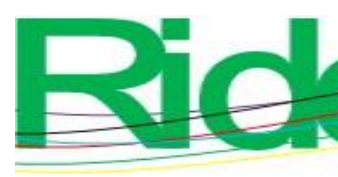

\section{Revista Iberoamericana para la Investigación y el Desarrollo Educativo ISSN 2007 - 7467}

Para J. Delors (1996), la educación, para cumplir con el conjunto de misiones que la sociedad le encomienda, debe estructurarse en torno a cuatro pilares: aprender a conocer, aprender a hacer, aprender a vivir juntos y aprender a ser. La Unesco (citada en Benito, 2011) afirma que es menester formar personas que ejerzan la ciudadanía plena y que participen activamente en procesos sociales. Además, promover "el fortalecimiento de las capacidades endógenas y la consolidación en un marco de justicia de los derechos humanos, el desarrollo sostenible, la democracia y la paz” (p. 231). Y por último:

Contribuir a proteger y consolidar los valores de la sociedad, velando por inculcar en los jóvenes los valores en que reposa la ciudadanía democrática y proporcionando perspectivas críticas y objetivas a fin de propiciar el debate sobre las opciones estratégicas y el fortalecimiento de opciones estratégicas y el fortalecimiento de enfoques humanistas (pp. 231-232).

Ahora bien, Bourdieu (1980) plantea la idea de que es posible conformar o configurar un habitus desde la puesta en liza de ciertas prácticas ciudadanas: a) responsabilidad democrática, $b$ ) valoración de las diferencias humanas y $c$ ) convivencia y paz (Cox, Bascopé, Castillo, Miranda y Bonhomme, 2014).

En tal sentido, los profesores de educación superior que recrean una cátedra o una práctica docente donde se aprecian las siguientes cualidades: a) conocimientos de la materia, $b$ ) habilidades o destrezas pedagógicas, $c$ ) relaciones interpersonales óptimas, $d$ ) características de una personalidad estable, $e$ ) relación de la docencia con la investigación y f) planificación docente, estarán gestando, decíamos, un habitus en los estudiantes ideal para el ejercicio de prácticas ciudadanas (Bourdieu, 2005).

Teniendo en cuenta lo anterior, en este trabajo se pretende identificar los atributos que caracterizan la práctica docente en educación superior y cómo se configura esta a partir de un paradigma de habitus ciudadano comprometido. Para ello, los objetivos del estudio son los siguientes:

- Identificar los atributos que caracterizan la práctica docente de maestros de la Unidad Académica de Psicología de la Universidad Autónoma de Zacatecas (UAZ).

- $\quad$ Reconocer el grado de ciudadanía en los estudiantes de educación superior de la Unidad Académica de Psicología de la UAZ respecto a la responsabilidad democrática, el respeto a las diferencias humanas y la convivencia y paz. 


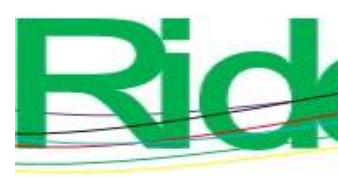

Revista Iberoamericana para la Investigación y el Desarrollo Educativo ISSN 2007 - 7467

- Correlacionar la práctica docente y las prácticas ciudadanas en estudiantes de educación superior de la Unidad Académica de Psicología de la UAZ.

\section{El paradigma de la práctica docente y la ciudadanía}

Existen complejas interacciones entre la cultura, el poder, la política y la educación. La práctica docente, configurada a partir de estilos personales, pero también de procesos de reformas producto de políticas educativas, está estrechamente ligada a los fenómenos sociales. La práctica docente de educación superior tiene un impacto preponderante y directo en la sociedad porque los egresados adultos son factores de cambio en su contexto, y llevan como parte de su capital social y cultural mucho de lo adquirido en las aulas. Justamente la relación educación superior y sociedad es motivo de análisis en estos momentos por tres razones fundamentales: el agotamiento del modelo educativo, la conformación de un nuevo marco social de referencia y el papel estratégico del conocimiento (Villaseñor, 1997). Lo anterior se explica a raíz de la crisis del proyecto moderno de educación y de sociedad gestado en el siglo XVIII en Occidente. Dicha crisis pone al sujeto en una condición posmoderna, lo cual significa que este está constituido como remanente de condiciones políticas, sociales y culturales que imponen una distancia que evita el cumplimiento de los objetivos de la modernidad, entre ellos, dos de los más importantes, la justicia y la igualdad.

La ciudadanía parece estar en boga, ser tema de discusión, porque lo está la democracia: existen preocupaciones por su debilitamiento y, a la vez, es vista como el único régimen político y de organización social hoy día aceptable. Asimismo, la preocupación se debe a que han aparecido nuevas circunstancias que afectan el marco político y cultural en el que venía siendo considerada (Gimeno, 2002). La erosión de los derechos sociales debido al fallido proyecto de la modernidad ha tenido como consecuencia que la posmodernidad como condición social se expanda y tenga así lugar una regresión del Estado del bienestar, sobre todo en las dos primeras décadas del siglo XXI. Las políticas económicas ahora neoliberales que han acentuado las desigualdades (trabajadores desempleados viviendo en pobreza o extrema pobreza, desatención a la educación y a la salud, cero contrataciones, abandono de los campesinos y los indígenas) nos han hecho más conscientes de la imperante necesidad de revitalizar los derechos de los ciudadanos (Meza, 2019). 


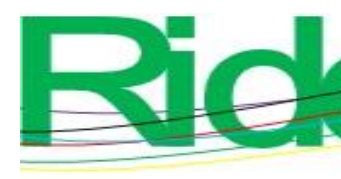

Revista Iberoamericana para la Investigación y el Desarrollo Educativo ISSN $2007-7467$

La crisis del proyecto neoliberal derivó en una descomposición social que afectó tanto a ciudadanos como a instituciones. Algunos de los síntomas de este proceso los expone Gimeno (2002):

El declive de la participación, la desconfianza y el desinterés de cada vez más individuos, ante el alejamiento de la política de los problemas que les afectan cotidianamente, deslegitima a la democracia como forma de gobierno de los asuntos públicos. La aparición de los movimientos sociales - como el feminista- resaltan reivindicaciones que denuncian el incumplimiento de los derechos básicos en grandes colectivos (p. 151).

Sin embargo, también se aprecia que la ciudadanía reclama una renovada atención por la desestabilización que están experimentando sus fundamentos ante realidades nuevas, unas veces, y otras ante problemas no adecuadamente resueltos (Gimeno, 2002).

Es fundamental que desde la escuela se funde la ideología de conducirse a partir de una libertad solidaria y no solitaria en la cual una constante sea la interdependencia, a partir de procesos sociales que limiten la mixofobia y promuevan la mixofilia (Bauman, 2017).

Para vivir juntos se requieren principios políticos más o menos compartidos y la ciudadanía permite legitimar una manera de ejercer la socialización en el seno de un Estado nación jurídicamente regulado. Bajo esta lógica, el individuo, en tanto ciudadano, puede gozar de ciertos "privilegios", como la igualdad, libertad, autonomía, justicia y derechos de participación (Gimeno, 2002). El interés de este estudio por el constructo de ciudadanía estriba en que implica la definición del individuo como sujeto y que, al mismo tiempo, permite verlo interdependiente, en relación con los demás, dando y recibiendo, lo cual a su vez implica una interacción al interior de los grupos donde se ponen en juego prácticas de liderazgo, toma de decisiones, comunicación y cohesión, elementos fundantes de la democracia.

El proyecto moderno, noble en sus principios, bajo la utopía de la premisa fundamental del progreso de la humanidad y de la emancipación de la ignorancia, delineaba una concepción de ciudadano educado que anteponía la razón al delirio y el derecho al abuso; en otras palabras, esbozaba una ciudadanía garante de igualdad. 


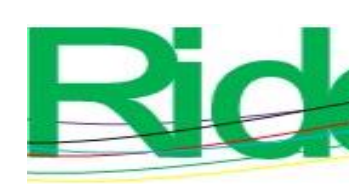

Revista Iberoamericana para la
Investigación y el Desarrollo Educativo
ISSN $2007-7467$

¿Qué significa esta cuando lo más evidente desde el punto de vista empírico son las desemejanzas entre ello? En la escuela, como en la vida y en la sociedad, la diferencia y la desigualdad son fácticas, pertenecen al reino de las realidades y de los hechos que nos vienen dados; la igualdad de la ciudadanía pertenece a la idealidad, es contrafáctica, un noble mito, como lo califica Heater (1999). Un imponente reto nos llama, pues: el de la reconstrucción de esa contrafacticidad para hacerla compatible con la idealidad. Ante esa misión se nos plantean algunas preguntas que nos inquietan: ¿Qué poder tiene la educación institucionalizada de invertir los hechos que en la sociedad real son contrarios al modelo real de ciudadanía? ¿Cómo hacerlo en lo que le sea factible actuar? ¿Qué significa la igualdad al lado de la libertad individual y de la fraternidad inherentes a la idea del ciudadano, cuando, sometidos a la evidencia de lo fáctico, vemos cómo se abandona la utopía de la igualdad? ¿Cómo entender las formas de la igualdad ante la evidencia de la diferencia y los valores de la individualidad? (Gimeno, 2002, pp. 153-154).

$\mathrm{Y}$ por supuesto, para responder las preguntas o inquietudes anteriores es preponderante revisar qué papel juega la práctica docente desde la universidad en la conformación de un sujeto con un habitus ciudadano.

\section{Práctica docente en educación superior. De la enseñanza tradicional a la conformación de sujetos educativos modernos}

Las instituciones educativas como dispositivos pedagógicos permiten construir formas de interacción e interdependencia entre el docente y los alumnos, así como entre alumnos y alumnos. Por consecuencia, constituye a los sujetos educativos, tanto al docente como al alumno, en la medida en que ocupan y hacen suyas las significaciones, normas y prácticas que el dispositivo, especialmente a través de la institución escolar, "instituye” para esos lugares (Foucault, 2002).

Los dispositivos actuales, modernos o posmodernos, exigen una práctica del docente universitario peculiar, a veces exigente y demandante, en el sentido de que solicitan del maestro lo siguiente: 


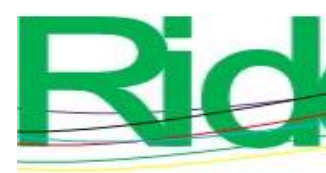

Revista Iberoamericana para la
Investigación y el Desarrollo Educativo
ISSN $2007-7467$

- $\quad$ Conocimiento sobre la materia.

- Habilidades o destrezas pedagógicas.

- Relaciones interpersonales.

- $\quad$ Características de personalidad.

- Relación docencia-investigación.

- $\quad$ Planificación docente (Prieto, Mijares y Llorent, 2015).

En cuanto a los modelos o paradigmas pedagógicos, se caracterizan por tres principios: ontológico, epistemológico y metodológico, lo cual delinea el conocimiento, la investigación y las prácticas en educación. Por lo tanto, todo modelo implica aspectos de orden discursivo y de orden práctico.

\section{Modelo tradicional}

Los procesos educativos giran en torno al docente. El alumno, por su parte, juega un papel pasivo. La forma de trabajo y de organización está basada en el magistrocentrismo (Abbagnano, 1994).

\section{Modelo escuela activa o escuela nueva}

En este modelo, el docente motiva la formación de un espíritu colectivo. Es el docente, por medio de ciertos dispositivos, y en conjunto con la escuela, que forman una cosmovisión que se edifica, necesariamente, en la relación social. El docente adecúa métodos y procedimientos de estudio a las características de los estudiantes. Ya no impera el reino de la prohibición para los alumnos, por lo tanto, pueden actuar con relativa libertad (Obregón, 2006).

\section{Modelo tecnocrático}

Está permeado por un paradigma de la psicología denominado conductismo. Este modelo representó una concepción tecnocrática aplicada a la enseñanza. Los elementos incipientes, en un principio, se fueron empleando inicialmente en la selección y adiestramiento del personal militar. Posteriormente, se fue articulando una propuesta educativa (Torres y Cobo, 2017). 


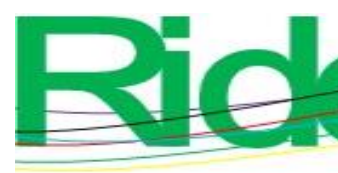

Revista Iberoamericana para la Investigación y el Desarrollo Educativo ISSN $2007-7467$

\section{La didáctica crítica}

Lo que es notable en este enfoque es la redimencionalización de los aspectos sociales de la enseñanza, ya que se considera que el aprendizaje meramente teórico es insuficiente. Se pone énfasis en la actividad social entre todos los agentes del hecho educativo, lo cual es una fuente rica en experiencias formativas (Apple, 2013).

\section{Modelo constructivista}

En el modelo constructivista confluyen varias teorías; tres de las más importantes son: la teoría psicogenética de Piaget, la teoría sociocultural de Vigotsky y la teoría cognoscitivista de Bruner y Ausubel (Hernández, 1998). El constructivismo, por lo tanto, es una unidad múltiple, ecléctica y, en ese sentido, compleja. Puede responder a las preguntas: ¿cómo se aprende?, ¿qué enseñar?, ¿cómo se debe enseñar?, ¿para qué enseñar determinado contenido?, ¿cuándo enseñar?, ¿qué, cuándo, cómo evaluar? No es una perspectiva que indique recetas infalibles, pero proporciona algunas orientaciones: a partir del nivel de desarrollo de los alumnos, asegurar la construcción de aprendizajes significativos.

La práctica docente es una actividad social que ejerce un profesional para dar clases, la cual está determinada por el contexto social, histórico e institucional. El ejercicio de la práctica es, pues, un factor importante en la educación: es el puente que permite conectar el proyecto educativo con el proyecto social. En esa línea de pensamiento, se asume que la práctica docente es un mecanismo que potencia que los estudiantes desde el aula vayan ejerciendo la ciudadanía desde sus tres esferas: participación democrática, respeto a la diversidad y convivencia y paz.

\section{Educación y ciudadanía}

Gimeno (2002) ha propuesto algunas razones que resultan fundamentales para relacionar la educación y la ciudadanía:

- Una de las primeras razones tiene que ver con el paralelismo que existe entre la capacidad creadora de la educación y de la ciudadanía democrática.

- Otra sería que la educación proporciona principios y valores coherentes con la ciudadanía. Hay una larga tradición emancipadora que constituyó una de las grandes narrativas de la educación, que tras una orientación democrática y con un impulso ético, vio a las escuelas como agentes para la mejora de la sociedad (Mora, 2012). 


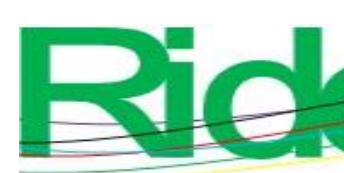

Revista Iberoamericana para la
Investigación y el Desarrollo Educativo
ISSN $2007-7467$

puesta en práctica de dichas variables); y la variable compleja de Ciudadanía se compone por las variables simples: "Responsabilidad democrática", "Valoración de las diferencias humanas" y "Convivencia y la paz" (19 reactivos evalúan la frecuencia y la puesta en práctica de dichas variables). Se utiliza la escala de medición de razón que va del 1 al 100. Es necesario destacar que para la elaboración de tablas y gráficos se recodificaron cada uno de los ítems en distintas variables con la finalidad de acotarlos aún más y, por lo tanto, simplificar y mejorar el análisis. De esta forma, se atribuyeron rangos de la siguiente manera:

- $\quad 1$ = Muy bajo, y aglutina el puntaje de 0 a 20 .

- $\quad 2$ = Bajo, y aglutina el puntaje de 21 a 40 .

- $\quad 3$ = Medio, y aglutina el puntaje de 41 a 60 .

- $\quad 4$ = Alto, y aglutina el puntaje de 61 a 80 .

- $\quad 5$ = Muy alto, y aglutina el puntaje de 81 a 100

La aplicación se llevó a cabo de manera grupal, en espacios escolares, y se pidió el consentimiento de cada uno de los participantes. Después de registrar los datos, se llevaron a cabo medidas de frecuencia, así como de correlación de Spearman.

\section{Resultados}

\section{Frecuencias de Práctica Docente}

A continuación, se presentan los análisis de frecuencia del componente Práctica Docente. A partir de la información obtenida en la tabla 1, se hace un cálculo de frecuencias de respuestas múltiples agrupando todas las variables del eje Prácticas Docentes ("Conocimiento sobre la materia", "Habilidades o destrezas pedagógicas", "Relaciones interpersonales", "Características de personalidad", "Relación docenciainvestigación" y "Planificación"). En esta se observa que $64.2 \%$ de los alumnos percibe a los docentes como académicos que llevan a cabo prácticas que demuestran que hay un "alto" y "muy alto" conocimiento sobre la materia, lo cual indica que se hace una apropiada selección de contenidos considerados como científicos, que propician que el educando adquiera conceptos (principios, hechos, leyes), procedimientos (habilidades y destrezas) y actitudes (valores). De igual forma, se entiende que la relación entre los contenidos y los niveles de complejidad son los apropiados para el nivel de licenciatura. En 


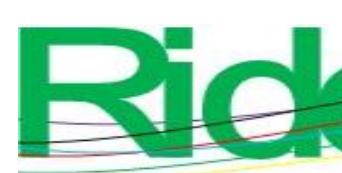

Revista Iberoamericana para la
Investigación y el Desarrollo Educativo
ISSN $2007-7467$

cuanto a la presentación que se hace a los alumnos, se destaca que el docente utiliza proyectos, problemas o centros de interés que son relevantes para los estudiantes.

Tabla 1. Práctica docente

\begin{tabular}{|c|c|c|}
\hline & & Respuestas \\
\hline & & Porcentaje \\
\hline Práctica & Muy bajo & $11.8 \%$ \\
\hline docente & Bajo & $7.3 \%$ \\
\hline & Medio & $16.8 \%$ \\
\hline & Alto & $32.2 \%$ \\
\hline & Muy Alto & $32.0 \%$ \\
\hline Total & & $100 \%$ \\
\hline
\end{tabular}

Fuente: Elaboración propia

Al referirnos a las habilidades o destrezas pedagógicas que posee el docente, $63.2 \%$ de los estudiantes señala que los docentes organizan y gestionan las actividades en el aula. Asimismo, orientan y dan sentido a los aprendizajes y promueven un clima socioemocional. Los docentes se preocupan por explorar y activar los conocimientos previos de los alumnos. Los estudiantes también mencionan que los docentes los apoyan en la profundización y elaboración de representaciones más complejas y expertas del contenido de enseñanza y aprendizaje.

En cuanto a la variable "Relaciones interpersonales", que tiene que ver con relaciones íntimas/superficiales, relaciones personales/sociales e incluso relaciones amorosas, $57 \%$ de los estudiantes señaló que las relaciones son superficiales, personales y se evita el acoso y hostigamiento entre docente-alumno.

Más de la mitad de los estudiantes encuestados (67\%) afirma que los docentes exhiben una personalidad con características orientadas hacia la extroversión, afabilidad, rectitud, estabilidad emocional y abierta a la experiencia, lo cual indica que las características de personalidad abonan a la buena práctica docente. Es, pues, un elemento importante a considerar como parte del análisis del quehacer docente.

La práctica docente relativa a la investigación está fortalecida, según más de $60 \%$ de los alumnos participantes, quienes expresan que los docentes ejercen su poder para ayudarlos, que los docentes analizan continuamente su proyecto de vida y profesional. Los 


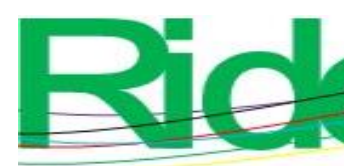

Revista Iberoamericana para la
Investigación y el Desarrollo Educativo ISSN $2007-7467$

estudiantes perciben que los docentes construyen su propio quehacer escolar con profesionalismo. Los docentes construyen teorías respetando el vientre cultural, edifican valores, le apuestan hacia la afectividad de sus alumnos, luchan contra la rutina de la práctica cotidiana. Los alumnos enfatizan que los forman con conciencia crítica. Se denota una intención por parte de los maestros por actualizarse y enriquecer su cátedra. Sin embargo, los estudiantes encuestados afirman que falta que los docentes formen con otros docentes investigadores grupos de pensamiento.

Respecto a la categoría "Planificación docente", 74 \% de los alumnos menciona que se alcanza a distinguir que sus maestros plantean objetivos, organizan los contenidos tanto conceptuales como procedimentales y actitudinales para hacerlos asequibles por medio de actividades didácticas e implementando algunos recursos (didácticos).

Los resultados de las frecuencias, en general, muestran que los estudiantes evalúan a la práctica docente de educación superior como muy buena y buena, $64.2 \%$ de los educandos así lo opinan.

Por otro lado, resulta interesante destacar que $35.8 \%$ de los jóvenes eligieron los valores "Muy bajo, "Bajo" y "Medio" para evaluar la práctica docente, lo cual indica que aún se puede fortalecer más en los docentes de educación superior los elementos que caracterizan la práctica docente en educación superior:

- $\quad$ Conocimiento sobre la materia.

- Habilidades o destrezas pedagógicas.

- Relaciones interpersonales.

- Características de personalidad.

- Relación docencia-investigación.

- Planificación docente.

Especialmente, las áreas de mayor oportunidad para los docentes tienen que ver con las variables "Docencia-investigación” y "Habilidades y destrezas pedagógicas", ya que en la medición de dichas variables los resultados se concentran entre el rango de "muy bajo", "bajo" y "medio". 


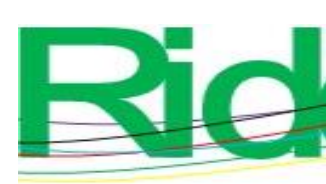

Revista Iberoamericana para la
Investigación y el Desarrollo Educativo
ISSN $2007-7467$

\section{Frecuencias de Ciudadanía}

En la tabla 2 se exhiben los resultados en porcentajes al agrupar todas las variables del eje Ciudadanía ("Responsabilidad democrática", "Valoración de las diferencias humanas" y "Convivencia y paz").

Tabla 2. Prácticas ciudadanas (Ciudadanía)

\begin{tabular}{|l|l|l|}
\hline \multicolumn{2}{|c|}{} & \multicolumn{2}{l}{ Respuestas } \\
\cline { 3 - 3 } & Porcentaje \\
\hline \multirow{3}{*}{$\begin{array}{l}\text { Prácticas } \\
\text { ciudadanas }\end{array}$} & Muy bajo & $15.3 \%$ \\
\cline { 2 - 3 } & Bajo & $7.4 \%$ \\
\cline { 2 - 3 } & Medio & $13.3 \%$ \\
\cline { 2 - 3 } & Alto & $23.1 \%$ \\
\cline { 2 - 3 } & Muy alto & $40.9 \%$ \\
\hline Total & $100.0 \%$ \\
\hline
\end{tabular}

Fuente: Elaboración propia

Se observa que $64 \%$ de los estudiantes muestra una "muy alta" y "alta" responsabilidad democrática. Por lo tanto, los alumnos participan tomando decisiones; tienen habilidades para potenciar discusiones y llegar a un acuerdo evitando que haya perdedores, de utilizar el voto para llegar a acuerdos.

En la variable compleja "Valoración de las diferencias humanas", 64 \% tiene una "muy alta" y "alta" habilidades para confrontar la discriminación por medio de mecanismos democráticos.

En la variable compleja "Convivencia y paz", 64 \% expone que posee "muy altas y "altas" habilidades para resolver pacíficamente situaciones como enfrentamientos, luchas, peleas o altercados. Igualmente, poseen habilidades para preservar, guardar, conservar y asistir a personas, seres vivos y medio ambiente. Este mismo $64 \%$ tiene una "muy altas" y "altas" habilidades emocionales para convivir, como la empatía, confianza y manejo de ira.

Aunque en general las prácticas de ciudadanía en estudiantes de educación superior son "altas" y "muy altas", es necesario destacar que $67.9 \%$ de los alumnos no toma decisiones en los grupos en los que participa. Además, $50 \%$ de los estudiantes no son percibidos como personas influyentes y generadoras de un mayor número de comunicaciones. Y 57.5\% no ha sido considerado como jefe de grupo tanto en los grupos 


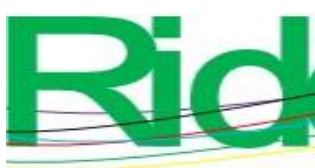

Revista Iberoamericana para la Investigación y el Desarrollo Educativo ISSN 2007 - 7467

\begin{tabular}{|c|c|c|}
\hline $\begin{array}{l}\text { 68) Consideras que tienes la capacidad y disposición para ser } \\
\text { portavoz a favor de otros. // 58) El docente determina si los } \\
\text { aprendizajes le han sido significativos y tienen sentido y valor } \\
\text { funcional. }\end{array}$ & $0.277 * *$ & 0.004 \\
\hline $\begin{array}{l}\text { 70) Consideras que tienes la capacidad y disposición para } \\
\text { confrontar comportamientos sociales que separen y consideren } \\
\text { inferiores a las personas por su raza, clase social, sexo, religión } \\
\text { u otros motivos ideológicos. // 44) El docente construye y vive } \\
\text { valores en su continuo indagar en los escenarios educativos y } \\
\text { sociales donde se desenvuelve. }\end{array}$ & $0.250^{* *}$ & 0.010 \\
\hline $\begin{array}{l}\text { 71) Cuando se produce un enfrentamiento, una pelea, una lucha } \\
\text { o una discusión, tienes la capacidad y disposición para resolver } \\
\text { de manera pacífica. // } 16) \text { Otorga sentido a la actividad } \\
\text { inmediata a partir de su relación con la actividad conjunta } \\
\text { anterior. }\end{array}$ & $0.282^{* *}$ & 0.003 \\
\hline
\end{tabular}

Fuente: Elaboración propia

En el análisis correlacional, se utilizaron todos los ítems del eje Práctica Docente y del eje Ciudadanía; los que aparecen en la tabla 3 son los que tienen un coeficiente de correlación positiva más alto.

A partir del análisis correlacional se pueden realizar las siguientes aseveraciones:

a) Cuando el docente analiza políticas, ideologías, paradigmas y enfoques para enriquecer su propio discurso y su propia práctica, los estudiantes se muestran al interior de sus grupos como las personas más influyentes y generadoras de un mayor número de comunicaciones.

b) Cuando el docente hace valoraciones de los procesos de enseñanza y aprendizaje mediante el diálogo entre los participantes del hecho educativo, los estudiantes manifiestan prácticas que los llevan a conducir a los grupos a los que pertenecen hacia sus metas.

c) Cuando el docente trabaja en equipo con sus pares, los estudiantes buscan recibir apoyo de los miembros de los grupos en los que participan y tienen la capacidad de influir de forma positiva. 


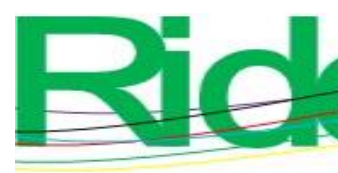

Revista Iberoamericana para la Investigación y el Desarrollo Educativo ISSN $2007-7467$

el campo educativo. Por lo tanto, la renovación de la práctica docente debe ir de la mano con las demandas del aprendizaje del siglo XXI.

Según Silva, Cristancho, Parra, Quevedo y Ródriguez (2015), pensar la ciudadanía solo como el ejercicio de la vida política sería limitar el concepto. Tradicionalmente, la ciudadanía definida como la participación política ha sido la concepción social predominante. De forma análoga a lo que Silva et al. (2015) exponen, el presente estudio exhorta a la comunidad educativa a construir nuevos significados de ciudadanía y ampliar su ámbito de aplicación, que ofrezca la visión de un ámbito público heterogéneo y de respeto y afirmación de las diferencias, y por extensión, reconocer las identidades y las diferencias, la justicia redistributiva y la participación democrática.

Los cambios profundos de civilización en los que estamos inmersos exigen que repensemos la educación y nos cuestionemos sobre cómo educar hoy a ciudadanos y ciudadanas que puedan y sepan ejercer sus derechos y no sean segregados o excluidos de la sociedad. Al igual que lo hace Valcárcel (2004), aquí también se precisa que es imperiosa y necesaria la vinculación de la democracia y la educación como requisito indispensable de la ciudadanía. Las instituciones educativas no pueden mantenerse al margen de las transformaciones sociales propias de la modernidad, neoliberalismo y la globalización. Las correlaciones abordadas en el apartado de resultados nos dan elementos para comprender cómo desde la práctica docente se puede lograr la conformación de una real ciudadanía.

Ahora bien, considerando las correlaciones realizadas, se encuentra una similitud con el estudio de Marí, Moreno e Hipólito (2016), quienes explican que la ciudadanía se adquiere de forma progresiva a través de su práctica:

- Implicarse en la formación y en asuntos públicos desde una edad temprana.

- La educación ciudadana es transversal al conjunto de contenidos educativos que se desarrollan.

- La práctica ciudadana favorece la inserción activa de las personas en diversos grupos e instituciones, participando en la vida grupal desde la individualidad y respetando la diversidad.

- Participar en formas democráticas de convivencia.

La educación ciudadana, tanto en educación superior como en los demás niveles educativos, no es responsabilidad exclusiva de los docentes (sin embargo, el papel que 


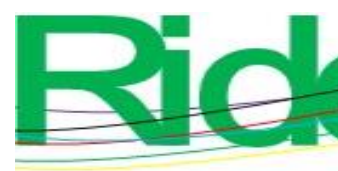

Revista Iberoamericana para la
Investigación y el Desarrollo Educativo
ISSN $2007-7467$

estos juegan es central), sino un compromiso desde la sociedad, la política y planes y programas de estudio.

En los datos obtenidos se identifica que los docentes implementan una praxis caracterizada por un paradigma de educación tradicional, ya que se refleja que el alumno juega un papel receptivo, el aprendizaje es memorístico y que el docente pretende ser el centro de los procesos áulicos (Hernández, 1998). Sin embargo, las políticas educativas actuales exigen que se elabore una práctica docente fundamentada en un modelo constructivista.

Una de las limitaciones de la investigación es que no se contempló un análisis de los principios curriculares de la educación superior, así como del propio currículo de la Unidad Académica de Psicología, esto hubiera permitido precisar cómo desde el “deber ser" se entiende la ciudadanía y la influencia que existe de los planes y programas de estudio con las prácticas docentes como con la formación ciudadana de los estudiantes.

Otra limitación es que no se consideró el punto de vista del profesorado para evaluar su propia práctica ni la opinión que tienen sobre los elementos de ciudadanía que pueden observarse al interior del centro educativo, esto coadyuvaría a hacer un contraste entre la perspectiva docente y del alumnado, y generar así una mayor riqueza de datos e información.

\section{Conclusiones}

La práctica docente permeada por $a$ ) conocimiento sobre la materia, $b$ ) habilidades o destrezas pedagógicas, $c$ ) relaciones interpersonales, $d$ ) características de personalidad, $e$ ) relación docencia-investigación y f) planificación está vinculada directamente con la responsabilidad democrática, la valoración de las diferencias humanas y la convivencia y la paz, es decir, con las prácticas de ciudadanía o con un habitus ciudadano de los alumnos de educación superior. A partir de la configuración y estructura que un docente elabore de su labor, instituye un sistema de prácticas de ciudadanía en los estudiantes de educación superior.

La figura 1 aborda las conclusiones más significativas, ya que sintetiza las variables simples, que son las más representativas de las categorías Práctica Docente y Ciudadanía. 
Figura 1. Interdependencia entre la práctica docente y la ciudadanía

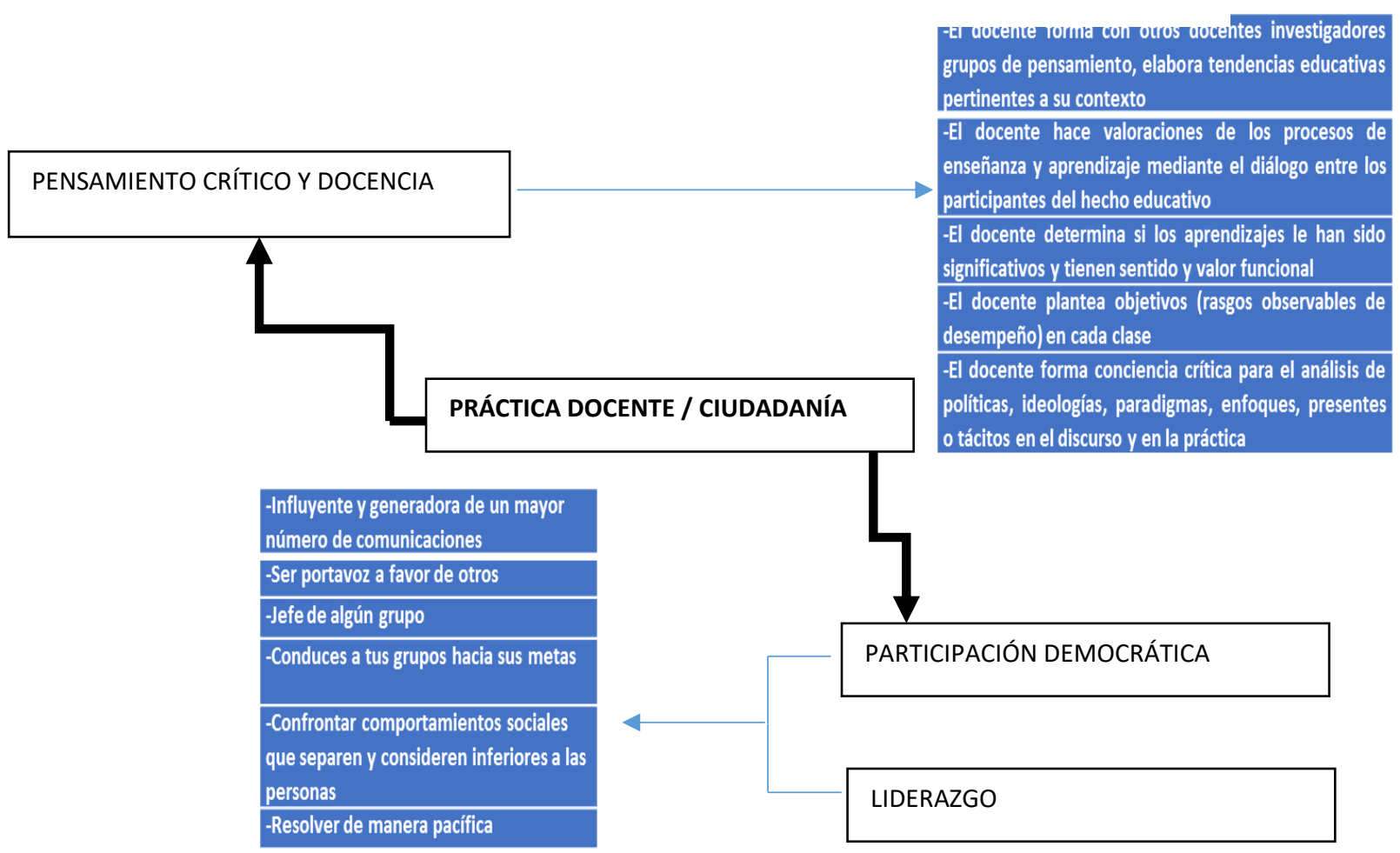

Fuente: Elaboración propia

A partir de la configuración y estructura de la labor docente desde los principios de un modelo ligado a la escuela nueva y constructivista, se instituye un sistema de prácticas de ciudadanía, las cuales se concretan con acciones de participación democrática y de liderazgo.

La variable "Pensamiento crítico y docencia", parte esencial en la práctica docente en la educación superior, se configura a partir de la coordinación real y eficaz con otros educadores y la búsqueda de la fidelidad a un proyecto común; el establecimiento de grupos de trabajo y la realización de valoraciones sobre la realidad escolar; la atención a la diversidad de capacidades, intereses y ritmos de aprendizaje, así como la comprobación del alcance y logro de los objetivos, desde una concepción holística y constructivista; el planteamiento de objetivos (rasgos observables de desempeño) en cada clase, y el análisis de políticas, ideologías, paradigmas y enfoques para generar precisamente un pensamiento crítico.

Cuando los docentes ponen en liza las variables anteriores, crean una estructura que permitirá que los estudiantes ejecuten prácticas de ciudadanía centradas en la participación democrática y el liderazgo, es decir, habrá elementos que permitirán ir estableciendo 

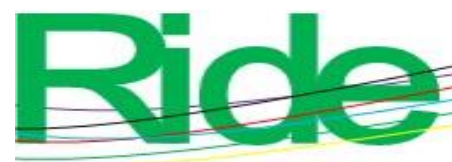

Revista Iberoamericana para la Investigación y el Desarrollo Educativo

ISSN $2007-7467$

condiciones desde la educación superior para que se forme un habitus ciudadano en los educandos, el cual se caracterizaría por las siguientes prácticas: el alumno influye y genera un mayor número de comunicaciones, es portavoz y está a favor de otros, se asume y juega el rol de responsable en algún grupo, conduce a su grupo hacia sus metas, confronta comportamientos sociales que separen y consideren inferiores a las personas y resuelve problemas de manera pacífica.

En general, en la muestra se observaron buenas prácticas de ciudadanía, sin embargo, falta fortalecer el liderazgo al interior de los grupos donde participan. Asimismo, las prácticas docentes de educación superior son buenas, pero se encuentra un área de oportunidad en la relación docencia-investigación y en las habilidades y destrezas pedagógicas.

Igualmente, destaca que dentro de la práctica docente el pensamiento crítico del profesor está estrechamente correlacionado con la conformación de este habitus ciudadano, pero específicamente con la participación democrática y el liderazgo. Es decir, los docentes de educación superior, al crear y recrear su práctica desde un pensamiento crítico, influyen directamente en la conformación de estudiantes ciudadanos que participan democráticamente y ejercen liderazgos en los grupos a los que pertenecen.

\section{Futuras Líneas de Investigación}

En las últimas décadas, debido a la internalización de la educación como respuesta a la globalización, una reestructuración curricular que retome los principios de la formación ciudadana se ha vuelto necesaria. Se debe poner énfasis en la relación que guarda el currículo de las instituciones de educación superior con las prácticas ciudadanas, pero específicamente con la participación democrática y el liderazgo político. 


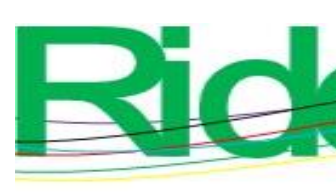

Revista Iberoamericana para la Investigación y el Desarrollo Educativo ISSN $2007-7467$

\section{Referencias}

Abbagnano, N. (1994). Historia de la filosofía (vol. 1). Barcelona, España: Hora.

Apple, M. W. (2013). Creando educación democrática en tiempos neoliberales y neoconservadores. Praxis Educativa, 17(2), 39-47. Recuperado de https://cerac.unlpam.edu.ar/index.php/praxis/article/view/777.

Bauman, Z. (2017). Sobre la educación en un mundo líquido. España: Paidós.

Benito, J. (2011). Ciudadanía, universidad y derechos humanos. Revista Electrónica Interuniversitaria de Formación del Profesorado, 14(1), 227-241. Recuperado de http://www.redalyc.org/articulo.oa?id=217017192018.

Bourdieu, P. (1980). Le capital social. Actes de la Recherche en Sciences Sociales, 31, 2-3.

Bourdieu, P. (2005). Intelectuales, política y poder (1. ${ }^{a}$ ed.). Buenos Aires, Argentina: Eudeba.

Cañedo, T. y Figueroa, I. E. (2013). La práctica docente en educación superior: una mirada hacia su complejidad. Revista Electrónica Sinéctica, 1(41), 1-18. Recuperado de https://www.redalyc.org/articulo.oa?id=99828325003.

Cox, C., Bascopé, M., Castillo, J. C., Miranda, D. y Bonhomme, M. (2014). Educación ciudadana en América Latina: Prioridades de los currículos escolares. IBE Working Papers on Curriculum Issues, (14). Recuperado de http://www.ibe.unesco.org/sites/default/files/resources/wpci-14education_ciudadana_spa.pdf.

Delors, J. (1996). La educación encierra un tesoro. Madrid, España: Santillana-Ediciones Unesco.

Foucault, M. (2002). Vigilar y castigar: nacimiento de la prisión. Buenos Aires, Argentina: Siglo XXI Editores.

Gimeno, J. (2002). Educar y convivir en la cultura global (2. ${ }^{a}$ ed.). Madrid, España: Ediciones Morata.

Hernández, G. (1998). Paradigmas en psicología de la educación. Ciudad de México, México: Paidós.

Marí, R., Moreno, R. e Hipólito, N. (2016). Educación y ciudadanía. Propuestas educativas desde la controversia. Foro de Educación, 14(20), 49-69. Recuperado de http://dx.doi.org/10.14516/fde.2016.014.020.005. 


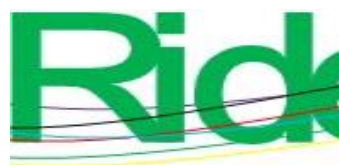

\section{Revista Iberoamericana para la Investigación y el Desarrollo Educativo ISSN 2007 - 7467}

Meza, E. (2019). Elecciones 2018 en México. Retorno al Estado de bienestar. Zacatecas, México: Universidad Autónoma de Zacatecas.

Mora, D. (2012). Formación democrática y escuelas democráticas para la construcción de ciudadanía crítica. Revista Integra Educativa, 5(2), 11-44. Recuperado de http://www.scielo.org.bo/scielo.php?script=sci_arttext\&pid=S19974043201200020 0002\&nrm $=$ iso.

Obregón, N. (2006). Quién fue María Montessori. Contribuciones desde Coatepec, 1(10), 149-171. Recuperado de https://www.redalyc.org/articulo.oa?id=28101007.

Organización de las Naciones Unidas para la Educación, la Ciencia y la Cultura [Unesco]. (2017). La formación inicial docente en educación para la ciudadanía en América Latina. París, Francia: Organización de las Naciones Unidas para la Educación, la Ciencia y la Cultura.

Prieto, M., Mijares, B. y Llorent, V. J. (2015). Cualidades del docente para la planificación curricular desde la perspectiva de los propios docentes y de sus estudiantes universitarios. REDU. Revista de Docencia Universitaria, 13(1), 157-179. Recuperado de https://polipapers.upv.es/index.php/REDU/article/view/6439/6504.

Silva, S., Cristancho, L., Parra, C., Quevedo, N. y Ródriguez, A. (2015). Ciudadanía juvenil: una breve revisión. Diversitas: Perspectivas en Psicología, 11(2), 273-288. Recuperado de https://www.redalyc.org/pdf/679/67944781008.pdf.

Torres, M., Yépez, D. y Lara, A. (2020). La reflexión de la práctica docente. Revista Chakiñan de Ciencias Sociales y Humanidades, (10), 1-18. Recuperado de https://www.redalyc.org/journal/5717/571763429006/571763429006.pdf.

Torres, P. C. y Cobo, J. (2017). Tecnología educativa y su papel en el logro de los fines de la educación. Educere, 21(68), 31-40. Recuperado de https://www.redalyc.org/articulo.oa?id=35652744004.

Valcárcel, A. (2004). La política de las mujeres. Madrid, España: Cátedra.

Villaseñor, G. (coord.) (1997). La identidad en la educación superior en México. Ciudad de México, México: Universidad Nacional Autónoma de México. 


\begin{tabular}{|c|c|}
\hline Rol de Contribución & Autor (es) \\
\hline Conceptualización & (David Jasso Velazquez «principal») \\
\hline Metodología & (David Jasso Velazquez «principal») \\
\hline Software & $\begin{array}{l}\text { (David Jasso Velazquez «principal»; Sonia Villagrán Rueda } \\
\text { «apoya») }\end{array}$ \\
\hline Validación & $\begin{array}{l}\text { (David Jasso Velazquez «principal»; Sonia Villagrán Rueda } \\
\text { «igual»; Mónica Rodríguez Ortiz «apoya») }\end{array}$ \\
\hline Análisis Formal & (David Jasso Velazquez «principal») \\
\hline Investigación & $\begin{array}{l}\text { (David Jasso Velazquez «principal»; Sonia Villagrán Rueda } \\
\text { «apoya»; Mónica Rodríguez Ortiz «apoya») }\end{array}$ \\
\hline Recursos & (David Jasso Velazquez «principal») \\
\hline Curación de datos & $\begin{array}{l}\text { (Sonia Villagrán Rueda «principal»; Mónica Rodríguez Ortiz } \\
\text { «apoya») }\end{array}$ \\
\hline $\begin{array}{l}\text { Escritura - Preparación del } \\
\text { borrador original }\end{array}$ & $\begin{array}{l}\text { (David Jasso Velazquez «principal»; Sonia Villagrán Rueda } \\
\text { «igual»; Mónica Rodríguez Ortiz «apoya») }\end{array}$ \\
\hline $\begin{array}{l}\text { Escritura - Revisión y } \\
\text { edición }\end{array}$ & $\begin{array}{l}\text { (David Jasso Velazquez «principal»; Sonia Villagrán Rueda } \\
\text { «igual»; Mónica Rodríguez Ortiz «apoya») }\end{array}$ \\
\hline Visualización & (Sonia Villagrán Rueda «principal») \\
\hline Supervisión & (David Jasso Velazquez «principal») \\
\hline Administración de Proyectos & (David Jasso Velazquez «principal») \\
\hline Adquisición de fondos & $\begin{array}{l}\text { (David Jasso Velazquez «principal»; Sonia Villagrán Rueda } \\
\text { «igual»; Mónica Rodríguez Ortiz «apoya») }\end{array}$ \\
\hline
\end{tabular}

